\title{
Ganglioneuroblastoma: Case report and review of the literature
}

\author{
S. Alessi ${ }^{a, *}$, M. Grignani ${ }^{b}$, L. Carone ${ }^{a}$ \\ ${ }^{a}$ Radiology Institute, San Matteo Policlinic, University of Pavia, Italy \\ ${ }^{\mathrm{b}}$ Department of Pediatrics, San Matteo Policlinic, University of Pavia, Italy
}

\section{KEYWORDS \\ Neuroblastoma; \\ Abdominal mass; \\ Ultrasonography.}

\begin{abstract}
Neuroblastoma are among the most important tumors of extracranial origin in pediatric patients. They arise from embryonal cells involved in the development of the sympathetic nervous system, whose differentiation has been arrested $[1,2]$. They are the tumors most frequently diagnosed during the first decade of life. Their evolution is unpredictable, ranging from progression to spontaneous regression or maturation, and their location and metastatic potential vary. Little is known about the cause of these tumors and the risk factors associated with their development. This article describes a typical case of ganglioneuroblastoma and provides a review of the literature on this type of tumor.
\end{abstract}

Sommario Il neuroblastoma è uno dei più importanti tumori pediatrici di derivazione extracranica; esso origina dalle cellule embrionali coinvolte nello sviluppo del sistema nervoso simpatico a causa di un blocco nel loro processo di differenziamento $[1,2]$. È la più frequente neoplasia della prima decade di vita; la sua progressione biologica è imprevedibile, regressione spontanea, maturazione a ganglioneuroma, localizzazione e metastatizzazione variabili. Poco è noto a riguardo dei fattori di rischio e della sua eziopatogenesi. Viene presentato un caso tipico di ganglioneuroblastoma e riesaminata la letteratura su questa neoplasia.

(c) 2011 Elsevier Srl. All rights reserved.

\section{Introduction}

Neuroblastic tumors account for $8-10 \%$ of all tumors diagnosed in pediatric patients and $80 \%$ of those found in children under 5 years of age [3]. They rarely develop in children over 10: the mean age at diagnosis in 22 months. Males are affected more frequently than females (ratio 1.2:1), and the incidence is higher in white children. The

\footnotetext{
* Corresponding author. Radiology Institute, San Matteo Policlinic, University of Pavia, Italy.

E-mail address: sarah.alessi@yahoo.it (S. Alessi).
}

tumors can arise wherever sympathetic nerve tissue is present. The most common locations include the adrenal glands (35\%), paraspinal retroperitoneal ganglia (30-35\%), posterior mediastinum (20\%), head and neck (1-5\%), and the pelvis (2-3\%) [4]; less often the tumor develops in the thymus, lung, kidney, anterior mediastinum, or cauda.

Around $1 \%$ of all neuroblastic tumors metastasize, generally via the vascular or lymphatic system. Common sites of metastatic involvement are the liver, lung, bone, and bone marrow. Patient age and tumor stage at diagnosis are major determinants of outcome: the prognosis for patients under 1 year of age is significantly better than that of older children with the same stage tumor. In the latter patients, there is a higher risk that the tumors will evolve into 
ganglioneuroblastomas. The clinical manifestations vary widely and are frequently nonspecific at the outset (asthenia, sweating, fever, pain related to the primary tumor or to metastatic lesions, and abdominal distension). Children under 2 years of age generally present with a large abdominal mass, fever, and weight loss. In older children, the cause may not be recognized until metastatic disease produces bone pain, respiratory problems, gastrointestinal disturbances, and the like.

Imaging studies play fundamental roles in the diagnosis and follow-up of neuroblastic tumors, and ultrasonography, which is easy to perform and does not involve radiation exposure, is the first-line method for both phases. Computed tomography and magnetic resonance imaging are the methods of choice when more accurate information is needed on the location of the disease, its relation to organs and vascular structures, and the presence of spinal involvement. Computed tomography is particularly important for evaluating the size of the mass and for identifying the organ of origin, infiltration of tissues and vascular structures, and the presence of adenopathy and calcifications, whereas MRI is the method of choice for assessing the extension of spinal tumors. These second-level studies are fundamental for planning surgery. Treatment options include surgery, chemotherapy, and radiotherapy. New
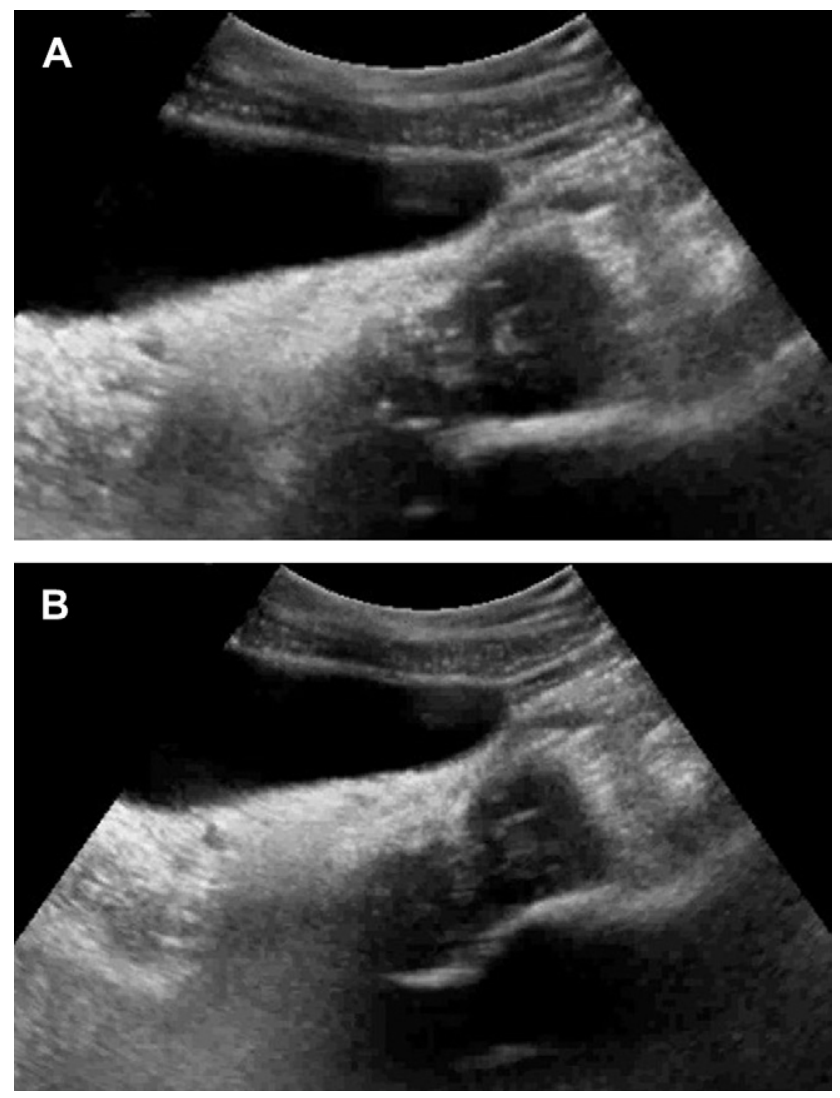

Fig. 1 Ganglioneuroblastoma in a 1-year-old boy: Ultrasonography performed after the patient had undergone surgery and two cycles of chemotherapy shows a persistent mass in the left iliac fossa. The residual disease is deep with respect to the bladder and exhibits an inhomogeneous echo structure with areas of calcification and necrosis.
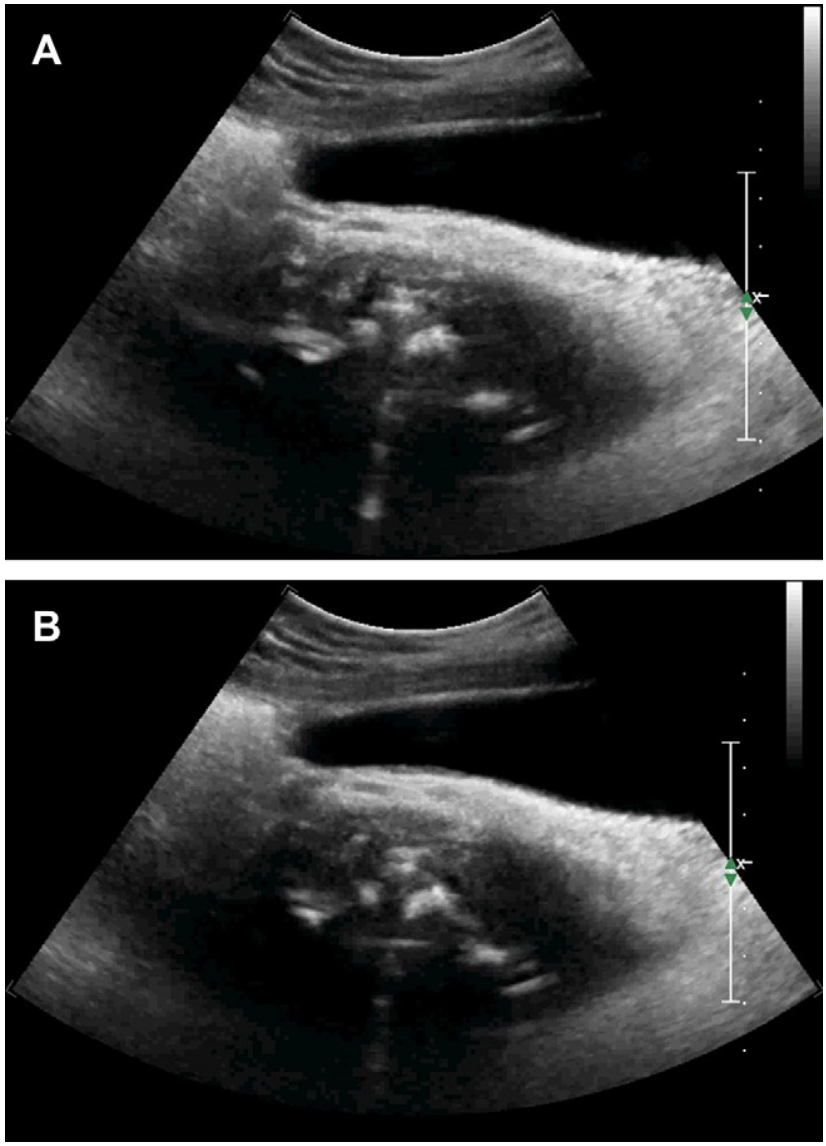

Fig. 2 Ganglioneuroblastoma in a 1-year-old boy: On followup sonograms obtained 3 and 9 months after surgery (panels $A$ and $B$, respectively), the residual mass with inhomogeneous echo structure remains unchanged.

prospects are emerging with the use of biological therapy based on the use of immunomodulators and retinoids.

The case described below is being reported with the informed consent of the patient's family.

\section{Case report}

The patient, a 1-year-old boy, was referred to the Department of Pediatric Oncology and Hematology in January

Table 1 International Neuroblastoma Pathology Classification (Shimada System).

\footnotetext{
- Stroma-poor neuroblastomas with neuroblasts that are undifferentiated, differentiating, or differentiated.

- Stroma-rich ganglioneuroblastomas with differentiated and undifferentiated neuroblasts.

- Stroma-dominant ganglioneuromas with maturing neuroblasts and mature ganglion cells.

- Composite nodular ganglioneuroblastomas with stroma-rich/stroma-dominant and stroma-poor cells and neuroblasts with various degrees of differentiation.
} 
Table 2 Prognosis of neuroblastic tumors classified according to Shimada system.

\begin{tabular}{|c|c|c|c|}
\hline International Neuroblastoma Classification & Patient Age (yrs) & Histology & Prognosis \\
\hline \multirow[t]{5}{*}{ Neuroblastoma (stroma-poor) } & $<1.5$ & $\begin{array}{l}\text { Poorly differentiated or differentiated \& } \\
\text { low or intermediate MKI tumor }\end{array}$ & Favorable \\
\hline & $1.5-5$ & Differentiated \& low-MKI tumor & Favorable \\
\hline & $<1.5$ & Undifferentiated low-MKI tumor & Unfavorable \\
\hline & $1.5-5$ & $\begin{array}{l}\text { Undifferentiated or poorly differentiated } \\
\text { tumor (regardless of } \mathrm{MKI} \text { ) }\end{array}$ & Unfavorable \\
\hline & $>5$ & All tumors & Unfavorable \\
\hline $\begin{array}{l}\text { Intermixed ganglioneuroblastoma } \\
\text { (stroma-rich) }\end{array}$ & All ages & All tumors & Favorable \\
\hline $\begin{array}{l}\text { Ganglioneuroma (maturing or mature; } \\
\text { stroma-dominant) }\end{array}$ & All ages & All tumors & Favorable \\
\hline $\begin{array}{l}\text { Ganglioneuroblastoma, nodular (stroma-rich, } \\
\text { stroma-poor, and stroma-dominant) }\end{array}$ & All ages & All tumors & Unfavorable \\
\hline
\end{tabular}

2003 for a 1-month history of high fever associated with sweating, moderate leukocytosis and thrombocytosis, and a palpable mass in the left iliac fossa. Abdominal sonography was done with a Philips IU22 scanner and a convexarray multifrequency transducer $(5-2 \mathrm{MHz})$. In correspondence with the palpable mass in the left iliac fossa, the scan revealed an oval-shaped mass with an inhomogeneous echo structure consisting of hypo- and anechogenic areas, portions of which were calcified. In preparation for surgery, an abdominal CT was done with a 16-detector scanner (Somatom Sensation - Siemens). Images were acquired with and without contrast enhancement. The examination confirmed the presence of a roundish mass (axial diameters: $4 \mathrm{~cm} \times 4 \mathrm{~cm}$; craniocaudal diameter $4.5 \mathrm{~cm}$ ) with well-defined margins, calcifications, and inhomogeneous enhancement after administration of the contrast agent. The anterior and posterior aspects of the mass were contiguous with the bones of the pelvis and spine, respectively. Given the tumor's proximity to the perivertebral nerves and its extensive calcification, the most likely hypothesis was neuroblastoma.

Surgery was performed in February 2003. Complete excision of the abdominal mass was precluded by involvement of adjacent pelvic nerves and blood vessels. Gross residual tumor was estimated at $20 \%$. Based on the pathology report, the diagnosis was intermixed ganglioneuroblastoma (Shimada classification: neuroblastic component $<50 \%$, (ow mitotic index) with no evidence of metastases.

The child later underwent two cycles of chemotherapy and was followed with regular clinical and imaging assessments. Since late 2004, he has been seen every three months. Each visit includes blood chemistry studies and abdominal imaging (ultrasonography (Fig. 1) and/or CT). MIBG scintigraphy has been consistently negative, and abdominal ultrasonography has shown residual tumor in the pelvis.

In 2006 enlargement of the pelvic mass was seen on ultrasound and confirmed on CT. In June 2007, laparotomy was performed, and the mass was biopsied. The specimen examined was characterized by morphologic and immunohistochemical features compatible with recurrent ganglioneuroma, maturing subtype. Considering the evidence of tumor-cell maturation, additional chemotherapy was deferred, and radical excision was precluded by the location of the mass and its relation to adjacent structures. The fact that

Table 3 The International Neuroblastoma Staging System.

Stage 1:

Localized tumor with complete gross excision, no gross or microscopic residual disease, and negative representative lymph nodes

Stage 2:

2A. Tumor with incomplete gross excision and negative representative ipsilateral lymph nodes. 2B. Tumor with incomplete gross excision, positive ipsilateral lymph nodes, and negative controlateral lymph nodes.

(Stage $1+$ Stage $2 \mathrm{~A}+$ Stage $2 \mathrm{~B}=25 \%$ )

Stage 3:

Unresectable tumor infiltrating across the midline, with or without regional lymph node involvement; or midline tumor with bilateral extension consisting of infiltration or lymph node involvement.

Stage 4:

Any primary tumor with dissemination to distant lymph nodes, bone, bone marrow, liver, or skin. (Stage $3+$ Stage $4=65 \%$ )

Stage 4S ("special" - 10\%): Localized primary tumor (as in stage 1, 2A, or 2B) with dissemination limited to skin, liver, and/or bone marrow (no bone involvement) in infants younger than 1 year. These infants have good adrenal function and more favorable prognoses. 
Table 4 Risk stratification based on prognostic factors.

High-risk tumors: Includes disseminated disease with multiple gene alterations including amplification of MYC-N (Corresponds to Stages 3 and 4). These tumors have the worst prognosis with cure rates below $20 \%$. Intermediate-risk tumors: Extensive disease with multiple gene alterations including amplification of MYC-N. These tumors progress slowly and are associated with cure rates of $25-50 \%$.

Low-risk tumors: Small tumors that can be treated with surgery alone (Stage 1 or $2^{\circ}$ ). These tumors are associated with cure rates exceeding $90 \%$.

the tumor was undergoing maturation suggested that the disease might well remain stable or even regress.

The patient still required close clinical and imaging follow-up every 1-3 months (Fig. 2) for at least 5 years. He is currently being followed regularly by our staff with clinical, laboratory, and imaging assessments.

\section{Discussion and conclusions}

Neuroblastic tumors arise from undifferentiated embryonal cells of the neural crest as a result of their arrested differentiation.

Most of these tumors are sporadic, but in $1-2 \%$ of all cases there is a family history of neuroblastic tumors characterized by autosomal dominant Mendelian transmission and incomplete penetrance.

Ganglioneuromas, ganglioneuroblastomas, and neuroblastomas differ from one another in terms of the stage of neuroblast maturation. The ganglioneuromas, which are composed of mature ganglion cells, are considered benign tumors. Ganglioneuroblastomas are less mature forms. They are regarded as more aggressive tumors, and they generally develop in small children (mean age at onset: 2 years). They tend to produce catecholamines, in particular vanillylmandelic acid and homovanillic acids, which are important diagnostic markers. For patients with ganglioneuroblastomas, the prognosis is relatively good: these tumors may regress spontaneously or mature into ganglioneuromas. Regression occurs in $1-2 \%$ of all cases, and the causes are unknown.

Neuroblastomas can produce metastatic disease via blood or lymphatic dissemination: those most common targets are the liver, the lungs, the bones, and the bone marrow. Children under 1 year of age often develop multiple skin metastases associated with bluish discoloration of the skin.

Neuroblastomas are currently defined as a heterogeneous group of tumors with biological properties that are closely correlated with their individual clinical and histological features. The International Neuroblastoma Pathology Classification is a revised version of the Shimada classification based on patient age at diagnosis, the abundance of stroma, and the stage of differentiation (Table 1). The International Classification introduces a prognostic index based on the numbers of cells undergoing mitosis and karyorrhexis
(MK index or MKI). Table 2 summarizes the characteristics of the various types of neuroblastic tumors, including age at diagnosis, histologic features, and prognosis (Shimada et al.) [5]. Diverse systems have been proposed for the staging of neuroblastomas. The stage of the tumor classified according to the International Neuroblastoma Staging System (INSS) (Table 3 ) is a major determinant of prognosis [6]. The tumors can also be divided into risk categories on the basis of prognostic factors (Table 4) [7].

Ultrasonography is the first-line method for identifying the tumor, which presents as a mass with inhomogeneous echo structure and areas of calcification and necrosis. CT and MRI are the imaging studies of choice for diagnosing ganglioneuromas and ganglioneuroblastomas. MRI is the preferred method for assessing the extension of spinal tumors, whereas CT is fundamental for estimating the size of the mass and for identifying the organ of origin, infiltration of tissues and vascular structures, and the presence of adenopathy and calcifications. Measurement of catecholamine metabolites in the urine and histological examination of biopsy specimens allow definitive diagnosis.

The case presented here confirms reports in the literature on the sensitivity and usefulness of ultrasonography as a first-level examination in children with suspected neuroblastic tumors, and it is the method of choice for follow-up and postoperative assessments of these young patients. The peculiarity of these tumors consists in their unpredictable biological evolution, which includes the possibility of spontaneous or treatment-induced differentiation of neuroblasts into mature ganglion cells, and the variability of their clinical and prognostic behavior, which often reflect their degree of differentiation.

\section{Conflicts of interest statement}

The authors have no conflicts of interest.

\section{Appendix \\ Supplementary material}

The supplementary data associated with this article can be found in the on-line version at doi:10.1016/j.jus. 2011.04.006.

\section{References}

[1] Medical Encyclopedia Medline Plus U.S. National library of Medicine and the National institutes of Health.

[2] Park JR, Eggert A, Caron H. Neuroblastoma: biology, prognosis, and treatment. Pediatr Clin North Am 2008;55(1):97-120.

[3] Kim S, Chung DH. Pediatric solid malignancies: neuroblastoma and Wilms' tumor. Surg Clin North Am Apr 1 2006;86(2):469-87.

[4] Aydn GB, Kutluk MT, Yalçn B, Büyükpamukçu M, Kale G, Varan A, et al. Neuroblastoma in Turkish children: experience of a single center. J Pediatr Hematol Oncol 2009 Jul;31(7):471-80.

[5] Shimada H, Ambros IM, Dehner LP, Hata J, Joshi VV, Roald B, et al. The International Neuroblastoma Pathology Classification (the Shimada system). Cancer 1999 Jul 15;86(2):364-72. 
[6] Brodeur GM, Pritchard J, Berthold F, Carlsen NL, Castel V, Castelberry RP, et al. Revisions of the international criteria for neuroblastoma diagnosis, staging, and response to treatment. J Clin Oncol 1993 Aug;11(8):1466-77.
[7] Look AT, Hayes FA, Shuster JJ, Douglass EC, Castleberry RP, Bowman LC, et al. Clinical relevance of tumor cell ploidy and $\mathrm{N}$-myc gene amplification in childhood neuroblastoma: a Pediatric Oncology Group study. J Clin Oncol 1991;9(4):581-91. 